\title{
A NEW PROOF OF A WEIGHTED INEQUALITY FOR THE ERGODIC MAXIMAL FUNCTION
}

\author{
KENNETH F. ANDERSEN ${ }^{1}$
}

\begin{abstract}
E. Atencia and A. de la Torre proved that the ergodic maximal function operator is bounded on $L^{p}(\omega)$ if $\omega$ satisfies an appropriate analogue of Muckenhoupt's $A_{p}$ condition. An alternate proof of this result is given.
\end{abstract}

Let $(\Omega, \Sigma, \mu)$ be a probability space and suppose $T: \Omega \rightarrow \Omega$ is an ergodic, invertible measure preserving transformation. If $\sigma \in L^{1}(d \mu)$ with $\sigma>0$ a.e., the two-sided ergodic maximal operator with respect to $\sigma$, denoted $M_{\sigma}$, is defined for nonnegative integrable $f$ by $\left(M_{\sigma} f\right)(x)=\sup _{m, n \geqslant 0} A_{\sigma}(f ; m, n, x)$ where

$$
A_{\sigma}(f ; m, n, x)=\frac{\sum_{k=-m}^{n} f\left(T^{k} x\right) \sigma\left(T^{k} x\right)}{\sum_{k=-m}^{n} \sigma\left(T^{k} x\right)}, \quad x \in \Omega .
$$

The one-sided maximal operators $M_{\sigma}^{+}$and $M_{\sigma}^{-}$are defined similarly except that the supremum is taken over $m=0, n \geqslant 0$ for $M_{\sigma}^{+}$and $m \geqslant 0, n=0$ for $M_{\sigma}^{-}$. If $\sigma$ is the constant function equal to one, these operators will be denoted simply as $M, M^{+}$ and $M^{-}$, respectively. Note that

$$
\left[\left(M_{\sigma}^{+}+M_{\sigma}^{-}\right) f\right] / 2 \leqslant M_{\sigma} f \leqslant\left(M_{\sigma}^{+}+M_{\sigma}^{-}\right) f .
$$

E. Atencia and A. de la Torre [1] proved that $M$ is bounded on $L^{p}(\omega d \mu)$, $1<p<\infty$, if $\omega \in L^{1}(d \mu)$ with $\omega>0$ a.e. and

$$
\left(\mathrm{A}_{p}\right) \quad\left[\frac{1}{i} \sum_{k=0}^{i-1} \omega\left(T^{k} x\right)\right]\left[\frac{1}{i} \sum_{k=0}^{i-1} \omega^{-1 /(p-1)}\left(T^{k} x\right)\right]^{p-1} \leqslant C \quad \text { a.e. }
$$

for some constant $C$ and all positive integers $i$. In this note an alternate proof of this result is given. Our proof, an adaptation of that given by $M$. Christ and $R$. Fefferman [2] for the Hardy-Littlewood maximal function operator, uses only elementary consequences of the $\left(A_{p}\right)$ condition; in particular, use of the "reverse Hölder" inequality property is avoided.

Received by the editors July 16, 1985.

1980 Mathematics Subject Classification. Primary 28D05; Secondary 42B25.

Key words and phrases. Maximal functions, ergodic maximal function, weighted inequalities.

${ }^{1}$ Research supported in part by NSERC grant A-8185. 
The Maximal Ergodic Theorem asserts that $M^{+}$is of weak type $(1,1)$ with respect to $d \mu$. The elegant proof of this result given recently by $\mathbf{R}$. Jones [3] is easily generalized to show that $M_{\sigma}^{+}$is of weak type $(1,1)$ with respect to the measure $\sigma d \mu$; indeed,

$$
\int_{\left\{x:\left(M_{\sigma}^{+} f\right)(x)>\lambda\right\}} \sigma(x) d \mu(x) \leqslant \frac{1}{\lambda} \int_{\Omega} f(x) \sigma(x) d \mu(x) .
$$

Since $T^{-1}$ is also ergodic and measure preserving, it follows that $M_{\sigma}^{-}$is also of weak type $(1,1)$ with respect to $\sigma d \mu$. Since these operators are clearly of strong type $(\infty, \infty)$, the Marcinkiewicz interpolation theorem shows that these operators are bounded on $L^{p}(\sigma d \mu), 1<p<\infty$; from (1) it follows that the same is true of $M_{\sigma}$.

Set $\sigma(x)=\omega(x)^{-1 /(p-1)}$. Observe first that if $\omega$ satisfies $\left(\mathrm{A}_{p}\right)$ then $\sigma \in L^{1}(d \mu)$. To see this, let $\sigma_{n}(x)=\min [\sigma(x), n]$ so that $\sigma_{n} \in L^{1}(d \mu)$ and $\left(\mathrm{A}_{p}\right)$ shows that

$$
\left[\frac{1}{i} \sum_{k=0}^{i-1} \omega\left(T^{k} x\right)\right]\left[\frac{1}{i} \sum_{k=0}^{i-1} \sigma_{n}\left(T^{k} x\right)\right]^{p-1} \leqslant C \quad \text { a.e. }
$$

The Dominated Ergodic and Monotone Convergence Theorems show, upon letting $i \rightarrow \infty$, then $n \rightarrow \infty$, that

$$
\left[\int_{\Omega} \omega d \mu\right]\left[\int_{\Omega} \sigma d \mu\right]^{p-1} \leqslant C .
$$

The main step in the proof is the estimate

$$
\int_{\Omega}\left(M^{+} f\right)^{p} \omega d \mu \leqslant B \int_{\Omega} f^{p} \omega d \mu
$$

for if this is proved, then upon replacing $T$ by $T^{-1}$ we obtain a similar estimate for $M^{-}$, and (1) then yields the required estimate for $M$.

Without loss of generality assume that $\int_{\Omega} f d \mu=1$ and then set $E^{k}=\{x \in \Omega$ : $\left.\left(M^{+} f\right)(x)>4^{k}\right\}$ for $k=1,2, \ldots$ Now (2) shows that $\mu\left(E^{k}\right)<1$ and since $T$ is ergodic it follows that for almost all $x \in E^{k}$ there are positive integers $r=r(x)$ and $s=s(x)$ such that $T^{j} x \in E^{k}$ if $-r+1 \leqslant j \leqslant s-1$ but $T^{j} x \notin E^{k}$ for $j=-r$ and $j=s$. Thus, if $B_{i}^{k}=\left\{x \in E^{k}: r(x)=1\right.$ and $\left.s(x)=i\right\}$, then the sets $T^{j}\left(B_{i}^{k}\right)$, $0 \leqslant j \leqslant i-1, i=1,2, \ldots$, are pairwise disjoint and their union (up to a set of measure zero) is $E^{k}$.

I wish to thank the referee for pointing out that this decomposition of $E^{k}$ is the same as that which results from the Kakutani decomposition of $\Omega \backslash E^{k}$ and in that context the inequalities (5) below were obtained by R. Jones [4].

We need the following lemma but postpone its proof until the end of this paper:

LEMMA. If $\chi_{k}$ denotes the characteristic function of $\Omega \backslash E^{k}$, then for $x \in B_{i}^{k}$

$$
4^{k}<\frac{1}{i} \sum_{j=0}^{i-1} f\left(T^{j} x\right) \leqslant 2 \cdot 4^{k}
$$


and

$$
\sum_{j=0}^{i-1} \sigma\left(T^{j} x\right) \leqslant\left(2^{p} C\right)^{1 /(p-1)} \sum_{j=0}^{i-1}\left(\sigma \chi_{k+1}\right)\left(T^{j} x\right)
$$

Now to obtain (4), write

$$
\int_{\Omega}\left(M^{+} f\right)^{p} \omega d \mu \leqslant 4^{p} \int_{\Omega} \omega d \mu+\sum_{k=1}^{\infty} 4^{(k+1) p} \int_{E^{k} \backslash E^{k+1}} \omega d \mu
$$

and observe that the first term on the right has the required bound, in view of (3), since by Hölder's inequality

$$
\int_{\Omega} \omega d \mu=\left[\int_{\Omega} \omega d \mu\right]\left[\int_{\Omega} f d \mu\right]^{p} \leqslant\left[\int_{\Omega} \omega d \mu\right]\left[\int_{\Omega} \sigma d \mu\right]^{p-1}\left[\int_{\Omega} f^{p} \omega d \mu\right] .
$$

On the other hand,

$$
4^{k p} \int_{E^{k} \backslash E^{k+1}} \omega d \mu \leqslant 4^{k p} \int_{E^{k}} \omega d \mu=\sum_{i=1}^{\infty} \int_{B_{i}^{k}} 4^{k p} \sum_{j=0}^{i-1} \omega\left(T^{j} x\right) d \mu
$$

and (5) shows that this is bounded by

$$
\begin{aligned}
\sum_{i=1}^{\infty} \int_{B_{i}^{k}}\left[\frac{1}{i} \sum_{j=0}^{i-1} f\left(T^{j} x\right)\right]^{p}\left[\sum_{j=0}^{i-1} \omega\left(T^{j} x\right)\right] d \mu \\
\quad=\sum_{i=1}^{\infty} \int_{B_{i}^{k}}\left[A_{\sigma}\left(f \sigma^{-1} ; 0, i-1\right)\right]^{p}\left[\sum_{j=0}^{i-1} \omega\left(T^{j} x\right)\right]\left[\frac{1}{i} \sum_{j=0}^{i-1} \sigma\left(T^{j} x\right)\right]^{p} d \mu .
\end{aligned}
$$

Now $\left(A_{p}\right)$ and (6) show that this is bounded by

$$
\begin{gathered}
(2 C)^{p^{\prime}} \sum_{i=1}^{\infty} \int_{B_{i}^{k}}\left[A_{\sigma}\left(f \sigma^{-1} ; 0, i-1\right)\right]^{p}\left[\sum_{j=0}^{i-1}\left(\sigma \chi_{k+1}\right)\left(T^{j} x\right)\right] d \mu \\
\leqslant(2 C)^{p^{\prime}} \sum_{i=1}^{\infty} \int_{B_{i}^{k}} \sum_{j=0}^{i-1}\left[\left(M_{\sigma} f \sigma^{-1}\right)\right]^{p}\left(\sigma \chi_{k+1}\right)\left(T^{j} x\right) d \mu \\
=(2 C)^{p^{\prime}} \int_{E^{k} \backslash E^{k+1}}\left[M_{\sigma} f \sigma^{-1}\right]^{p} \sigma d \mu .
\end{gathered}
$$

Summing over $k$ and using the boundedness of $M_{\sigma}$ on $L^{p}(\sigma d \mu)$ shows that the second term in (7) also has the required bound. Thus we have (4).

It remains only to prove the lemma.

The right-hand inequality of (5) is clear since $T^{-1} x \notin E^{k}$ implies

$$
\frac{1}{i+1} \sum_{j=-1}^{i-1} f\left(T^{j} x\right) \leqslant 4^{k}
$$

Since $x \in E^{k}$ there is a positive integer $n$ such that

$$
\sum_{j=0}^{n-1} f\left(T^{j} x\right)>4^{k} n
$$


We may assume that $n \geqslant i$, for otherwise, to derive a contradiction, let $n$ be the largest integer satisfying (8). Then, since $n<i, T^{n} x \in E^{k}$ so there is a positive integer $m$ such that $\sum_{j=n}^{n+m-1} f\left(T^{j} x\right)>4^{k} m$. Adding this to (8) then contradicts the maximality of $n$. On the other hand, if $n>i$ in (8), then $T^{i} x \notin E^{k}$ implies

$$
\sum_{j=i}^{n-1} f\left(T^{j} x\right) \leqslant 4^{k}(n-i) \text {. }
$$

Subtracting this from (8) shows that (8) also holds for $n=i$. This proves (5).

Next we show that

$$
\sum_{j=0}^{i-1} \chi_{k+1}\left(T^{j} x\right)>i / 2, \quad x \in B_{i}^{k}
$$

Write $J=\left\{j: 0 \leqslant j \leqslant i-1, T^{j} x \in E^{k+1}\right\}$ as a disjoint union of maximal blocks of consecutive integers $J_{q}, q=1, \ldots, m$. If $|J|$ denotes the cardinal of $J$, then by (5) we have

$$
|J|=\sum_{q=1}^{m}\left|J_{q}\right|<\sum_{q=1}^{m}\left[4^{-k-1} \sum_{n \in J_{q}} f\left(T^{n} x\right)\right] \leqslant 4^{-k-1} \sum_{j=0}^{i-1} f\left(T^{j} x\right)
$$

and again by (5), this does not exceed $i / 2$. This proves (9).

The $\left(A_{p}\right)$ condition and $(9)$ show that

$$
\left[\sum_{j=0}^{i-1} \omega\left(T^{j} x\right)\right]\left[\sum_{j=0}^{i-1} \sigma\left(T^{j} x\right)\right]^{p-1} \leqslant C i^{p} \leqslant 2^{p} C\left[\sum_{j=0}^{i-1} \chi_{k+1}\left(T^{j} x\right)\right]^{p}
$$

and then Hölder's inequality shows that this does not exceed

$$
2^{p} C\left[\sum_{j=0}^{i-1}\left(\omega \chi_{k+1}\right)\left(T^{j} x\right)\right]\left[\sum_{j=0}^{i-1}\left(\sigma \chi_{k+1}\right)\left(T^{j} x\right)\right]^{p-1} \text {. }
$$

From this (6) follows and the lemma is proved.

\section{REFERENCES}

1. E. Atencia and A. de la Torre, $A$ dominated ergodic estimate for $L^{p}$ spaces with weights, Studia Math. 74 (1982), 35-47.

2. M. Christ and R. Fefferman, A note on weighted norm inequalities for the Hardy-Littlewood maximal operator, Proc. Amer. Math. Soc. 87 (1983), 447-448.

3. R. Jones, New proofs for the maximal ergodic theorem and the Hardy-Littlewood maximal theorem, Proc. Amer. Math. Soc. 87 (1983), 681-684.

4. , Inequalities for the ergodic maximal function, Studia Math. 60 (1977), 111-129.

Department of Mathematics, University of Alberta, Edmonton, Alberta, Canada 